\title{
Biological effectiveness of polyfunctional biopreparations in soft wheat cultivation and assessment of crop quality based on NDVI index
}

\author{
Irina I. Novikova ${ }^{1, *}$, Elza V. Popova ${ }^{1}$, Leonid E. Kolesnikov ${ }^{2}$, Nikolay S. Priyatkin ${ }^{3}$ \\ and Yulia R. Kolesnikova ${ }^{4}$
}

\author{
${ }^{1}$ All-Russian Institute of Plant Protection, Saint Petersburg, 196608, shosse Podbelskogo, 3, Russia \\ ${ }^{2}$ Saint-Petersburg State Agrarian University, Saint Petersburg, 196601, St. Petersburg shosse, 2 , \\ Russia \\ ${ }^{3}$ Agrophysical Research Institute, Saint Petersburg, 195220, Grazhdansky pr. 14, Russia \\ ${ }^{4}$ Federal Research Center N.I.Vavilov All-Russian Institute of Plant Genetic Resources, Saint \\ Petersburg, 190000, ul. Bolshaya Morskaya, d.42-44, Russia
}

\begin{abstract}
The influence of Bacillus subtilis strains and chitosan complexes on the soft wheat productivity and its infection with disease agents was determined in order to develop new preparative forms of polyfunctional biopreparations. The prospects of using polyfunctional preparations, combined the beneficial properties of microorganism strains as pathogen antagonists and chitosan as plant disease resistance activator has been shown the study. Their effectiveness has been convincingly demonstrated in protecting wheat from root rot, in increasing yields and improving grain quality Plants treated with complex preparation containing bacterial culture liquid ("Vitaplan, CL") or its concentrate ("Vitaplan, CCL") and Chitosan II had the best adaptive potential to agro-ecological environmental factors. At the same time, the «Vitaplan, CCL and Chitosan II» complex had the most pronounced protective properties. In the case of "Vitaplan, CL and Chitosan II» treatments, plants had the highest values of wheat potential (biological) yield. It was shown that the vegetative index NDVI, determined by the results of spectrometric analysis, can be used as an integral parameter of the biological effectiveness of polyfunctional biopreparations. The maximum value of NDVI was revealed in the case of "Vitaplan, CL and Chitosan II"
\end{abstract}

\section{Introduction}

One of the main trends of modern crop production is the use of plant protection agents and technologies facilitating effective control of harmful organisms and conversion to biological farming. For many years All-Russian Institute of Plant Protection (VIZR) has been successfully developing polyfunctional preparations, which combine direct inhibitory effect on target objects and a positive effect on plants (increased disease and stress resistance, yield,

\footnotetext{
*Corresponding author: irina_novikova@inbox.ru
} 
improved product quality). Currently, one of our priority research directions is the development of technologies for the production and application of new environmentally safe polyfunctional preparations combining beneficial properties of strains of phytopathogen antagonists and chitosan compositions - plant disease resistance activators.

The methodology for such formulations is based on the integration of two directions of research which have been carried out for many years in the laboratories of microbiological plant protection and phytotoxicology of VIZR $[1,2]$. The first direction is related to the development of a concept for the creation and application of polyfunctional biopreparations based on living cultures of microorganisms and complexes of their metabolites for the effective suppression of phytopathogens and phytosanitary optimization of agroecosystems. The basis of such biopreparations are the strains with high level of biological activities including fungicidal, bactericidal, antiviral and plant growth regulatory effects, further characterized by high productivity and safety for warm-blooded animals and humans. As the result of the research, a number of biopreparations authorized in the Russian Federation and successfully applied in agriculture has been developed [3].

The second direction is the development and application of chitosan composite preparations with signal molecules possessing high speed and intensity for inducing systemic protective reactions in plants. VIZR has developed the concept for creating chitosan-based preparations for plant protection [4]. Chitosan is used as an inductor of non-specific resistance in combination with signal molecules of different mechanism of action. The effectiveness of the preparations is $70-90 \%$, which is $20-30 \%$ higher than the original unmodified chitosan. Five patents, two of which are international, have been obtained for these preparations (Chitosar series).

In recent years, as a result of VIZR merging the two directions into one, a number of preparations of the Chitosar Bio series has been developed based on the immobilization of microorganism cells on chitosan carriers. The polymer structure allows combining in one preparation associations of microorganisms with different target biological activities (antagonists of pathogens, growth and development stimulants, nitrogen fixators and microorganisms involved in the mineralization of organic matter. Biological effectiveness of preparations against fusarious root rot of cucumber and tomato is $60.0-70.0 \%$. The preparations exhibit a pronounced plant growth regulatory effect. Patents have also been obtained for these preparations.

Recently, polyfunctional complexes, including the strains of microorganisms-antagonists of plant disease agents and the chitosan molecules with signal molecules, have been developed. As shown by our previous studies, the B. subtilis 2604D strain synthesizes a polypeptide antibiotic from the bacteriocin group and a polyene antibiotic, and the $B$. subtilis B-2605D strain forms a polypeptide close to bacillin and hexaene antibiotics, one of which belongs to the subgroup of mediocidine. Preparations of this type have complex action, significantly change the metabolism of plants and the features of pathogenesis in the parasitehost system [5]. Therefore, the search for integral indicators for assessing the biological effectiveness of such multifunctional preparations is of particular importance.

The aim of the research is to determine the effect of biopreparations and chitosan complexes on wheat productivity and the intensity of disease development in spring soft wheat.

\section{Materials and Methods}

Experimental research was carried out in the experimental field of Pushkin laboratories of the Federal research centre «N.I.Vavilov All-Russian Institute of Plant Genetic Resources» (VIR). The wheat cultivar, Leningradskaya 6, c-64900 (Russia, Leningrad region) had been 
used as the experimental sample for revealing the polyfunctional complexes effect on the wheat productivity and diseases intensity. This sample was provided for study by the VIR Wheat Genetic Resources Department.

Wheat seeds of Leningradskaya 6 k-64900 cultivar were treated before sowing, and its vegetative plants were sprayed three times by the preparations.

The experiment scheme included the following options:

1. Control (water)

2. Vitaplan wettable powder - WP (standard) containing cells of Bacillus subtilis B-2604D and B. subtilis B-2605D strains..

3. Vitaplan, CL - culture liquid of B. subtilis B-2604D strain and B.subtilis B-2605D strain at ratio $1: 1$ with titer of live cells $\left(10^{10} \mathrm{CFU} \mathrm{ml-}{ }^{1}\right)$ and $10^{10}$ spores/g of $B$. subtilis.

4. Polyfunctional complex: Vitaplan, CL and Chitosan II.

5. Polyfunctional complex: Vitaplan CCL (concentrate of cultural liquid) and Chitosan II.

Since our long-term studies have established the high biological effectiveness of Chitosan II in protecting soft wheat from a complex of major diseases and a positive effect on its productivity, in this study we used this drug only in combination with microorganisms [6].

Wheat spraying with the preparations was carried out in the phases of tillering, stem elongation, beginning of ear formation. Wheat productivity was studied in the phase of earing-flowering and maturation by a set of indicators characterizing the morphological characteristics of plants and the yield structure. In the earing-flowering phase, a complex of plant indicators was studied: the productive and general bushiness (pieces), the plant phase (digital code, Zadoks scale), the flag and pre-flag leaf area $\left(\mathrm{cm}^{2}\right)$, the height of plants $(\mathrm{cm})$, the length of spike $(\mathrm{cm})$, the number of spikelets per spike (pieces), the weight of spike $(\mathrm{g})$. In addition, we determined the number and length of roots (the main embryonic root, embryonic coleoptile and roots) extending from the epicotyl. The indices of root weight and vegetative part of plants weight were calculated. The sample size for each variant of the experiment was 10-15 plants, which provided a reliable result for measuring the complex of the above indicators at $\mathrm{P}>0.95$.

The maturation phase (phase of full ripeness) studied the structure of wheat yields by indicators: the number of spikelets per spike; the spike length, $\mathrm{cm}$; the spike weight; the number of grains per spike; the grain weight per spike; the 1000 grains weight. The potential (biological) yield of a single wheat plant was calculated in accordance with the values of the productive bushiness and the grain weight per spike of one plant (g/plant). The potential yield $\left(Y_{n}\right)$ of wheat varieties in relation to the area of sowing $(t / h a)$ was determined by the productive bushiness, the grain weight per spike and the number of plants sown per $1 \mathrm{~m}^{2}$ according to the original formula:

$$
Y_{\Pi}=M_{\mathrm{K}} \cdot \mathrm{K}_{\Pi} \cdot P_{\Pi} \cdot 10000
$$

Where are: $M_{k}$ - grain weight per spike $(t) ; K_{n}$ - productive bushiness of the sample; $P_{n}$ sowing density (number of plants per $1 \mathrm{~m}^{2}$ ). In estimation the grain weight per spike and the productive bushiness for each variant of the experiment the amount of sampling was 10-15 plants.

Assessment of the degree of damage to plants by root rot was carried out in laboratory conditions in the phase of finished tillering of wheat and earing-flowering according to the scale: 0-epicotyl without lesion, 1-single spots on epicotyl; 2- strong defeat; 3- fatal defeat, a plant is died. In each variant of the experiment, 20 plants were evaluated. The development of root rot according to the variants of the experiment was determined taking into account the weighted average of the degree of plant damage:

$$
R_{r}=\frac{\sum(\mathrm{a} \cdot b) \cdot 100}{\mathrm{~A} \cdot \mathrm{K}}
$$


Where are:

$\mathrm{R}_{\mathrm{r}}$ - development of root rot

a - number of plants with the same signs of damage;

$\mathrm{b}$ - the corresponding score;

A - number of plants registered (healthy and sick);

$\mathrm{K}$ - the highest score of the accounting scale.

The intensity of development of pathogens of wheat leaves was taken into account in the following phases and stages of wheat ontogenesis: the phase of tillering of wheat (finished tillering), the phase of flag sheet (the elongation, the end of elongation), the phase of earings (the end of the earings), phase of flowerings (the start of flowerings and the end of flowerings), maturation (milk ripeness of grain; wax ripeness). The intensity of the defeat of the flag and pre-flag leaves of wheat pathogen powdery mildew (Blumeria graminis Speer.) was assessed visually according to the degree of plant damage, as well as additional indicators - the number and area of powdery mildew stains.

The number of powdery mildew stains was determined by counting them, based on the area of the wheat leaf:

$$
S_{\text {п }}=0.7 \cdot L \cdot S
$$

where are: 0.7 is the narrowing factor, $\mathrm{L}$ - length of leaf $(\mathrm{cm}), \mathrm{S}$ - width of leaf $(\mathrm{cm})$.

When we registered a large number of spots with a touch of powdery mildew on the leaf, their number was first counted in $1 \mathrm{~cm}^{2}$ of the leaf, and then we transferred the value to the entire area of the wheat leaf. For each variant of the experiment the values of the above indicators of the pathogenesis of powdery mildew of wheat were determined by the results of the analysis of 15 flag and 15 pre-flag leaves. Defeat flag and pre-flag leaves of wheat pathogen brown rust Puccinia recondita Rob. ex Desm. f. sp. tritici Eriks. counted on the Peterson scale. The number of pustules per leaf, the area of the pustule were used as additional phytopathological parameters. The number of pustules was determined by counting them on wheat leaves using the MBS-10 microscope.

When a significant number of brown rust pustules were detected, their number per $1 \mathrm{~cm}^{2}$ of the sheet was first determined and then the value was recalculated to the entire surface area of the sheet. For each variant of the experiment the values of the above indicators pathogenesis of brown rust of wheat were determined by the results of the analysis of 15 flag and 15 pre-flag leaves.

The degree of damage of samples by wheat leaf blotch (Stagonospora nodorum Castell.et Germano) was determined according to the visual scale of James. For each variant of the experiment the intensity of Septoria development was characterized by the results of the analysis of 15 flag and 15 pre-flag leaves.

The intensity of wheat defeat by yellow rust was assessed on the Manners scale and, in addition, visually, with using a microscope MBS-10. The number of pustules (total per leaf), the yellow rust strips number with pustules, the length of strips with pustules, the area of the pustule and their number in the strip were used as the additional indicators of pathogenesis. For each variant of the experiment the values of the above indicators of yellow rust pathogenesis were determined by the analysis of 15 flag and 15 pre-flag leaves.

The size of the powdery mildew stains and rust's pustules (brown rust, yellow rust), which were formed in pathogenesis on wheat leaves, was determined using ocular and objective micrometers. The values of pustule area and powdery mildew stains (infectious structures $\mathrm{S}_{\mathrm{i} . \mathrm{s} .}$ were calculated under the assumption on their elliptic form using the expression:

$$
S_{i . S .}=m \cdot \pi \cdot a \cdot b
$$

Where: $a$ and $b$ - the values of the semiaxes of the ellipse (in the lines of the ocular micrometer), $\mathrm{m}$ - the scale factor of the microscope. 
The use of this pathogenesis indicators set allowed to expand the range of statistical analysis methods applicable to the study and to increase the accuracy of the experiment in determining the biological effectiveness of the polyfunctional complexes.

The optical characteristics of the crop phytosanitary state were determined by the GreenSeeker instrument based on the calculation of the NDVI (Normalized Difference Vegetation Index), which serves as a relative indicator of the amount of photosynthetically active biomass and allows to determine changes in the crops state accurately [7].

Statistical analysis of the results was carried out in the programs SPSS 21.0, Statistica 6.0, Excel 2016. The methods of parametric statistics were used in the calculations (based on mean $\mathrm{M}$ and their standard errors \pm SEM; and 95\% confidence intervals and Student's t-test).

The cluster analysis method of divisive strategy (k-means) was used for comparative analysis of relative changes in pathogenesis parameters in the application of biopreparations and chitosan complexes as compared to the control. The validation of cluster analysis results was performed by the dispersion analysis method. It should be noted that the more the Fcriterion value increases, the more the null hypothesis (H0) about the absence of the biological products and multifunctional complexes influence on the pathogenesis is violated.

\section{Results and Discussion}

Figure 1 presents field evaluation results of the effectiveness of biopreparations and chitosan complexes against root rots and the diseases of spring soft wheat leaves.

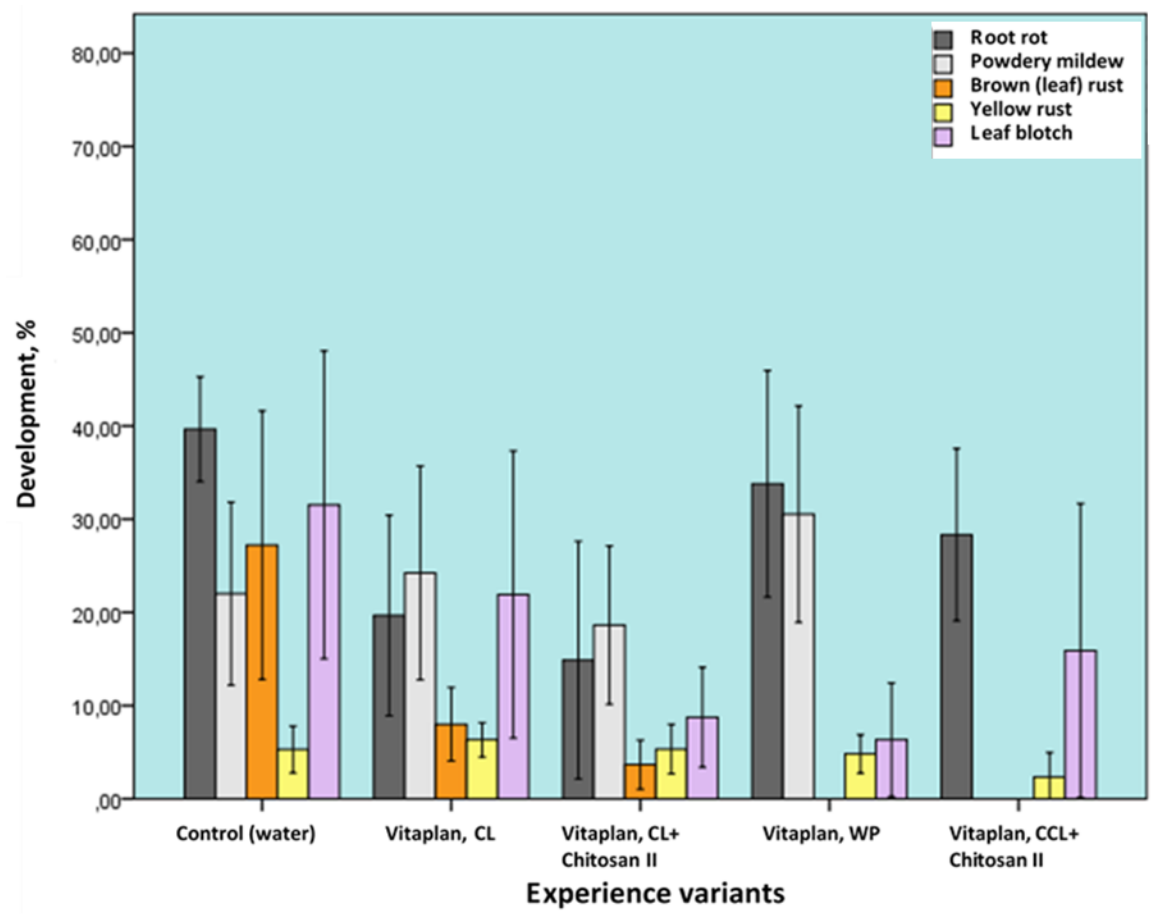

Fig. 1. Influence of biopreparations and polyfunctional complexes on the wheat disease development. (95\% confidence intervals for means). SPb. - Pushkin, 2016-2018. 
The Vitaplan, CL and Chitosan II polyfunctional complex treatments by the period of 2016-2018 (Fig.1) have resulted significant decrease of $\mathrm{p}<0.05$ in the intensity of the development of helmintosporiosis rot agents (by 25\%, student's t-test $=3,5$ ), brown rust on flag leaves (by $24 \%, t=3,1$ ) and wheat leaf blotch on pre-flag leaves (by $23 \%, t=2,6$ ).

In the case of Vitaplan, CCL and Chitosan II treatment, there was a significant decrease in the development of root rot (by 19\%, $\mathrm{t}=2,8$ ) and wheat leaf blotch (by $19 \%, \mathrm{t}=3,1$ ). There was no damage caused by brown rust and powdery mildew in these treatments.

By the method of cluster analysis 3 groups of pathogenesis indicators formed during the development of wheat disease agents were identified. These groups differed by the relative change towards the control according to the test variants. In general, the use of Fisher (F) criterion supported that the "Vitaplan, CCL and Chitosan II" polyfunctional complex reduced the intensity of disease agents' development to the greatest value among the tested variants. An important indicator of rust pathogenesis is the total number of pustules on a leaf. In the treatments using the "Vitaplan, CL, CCL and Chitosan II" polyfunctional complexes the total number of yellow rust pustules on the flag leaf was reduced by $50-60 \%$, and brown rust - up to $60 \%$. Mostly, the area of the empty brown rust decreased in the Vitaplan, CCL and Chitosan II (by 43\%) and the Vitaplan, CL treatments (by 54\%) as compared to the control

A significant decline in the development of major wheat diseases influenced its potential yield. In the case of Vitaplan, CL and Chitosan II, wheat yield increased on average by $68.7 \%$ as compared to the control over the period of 2016 -2018 (Figure 2).

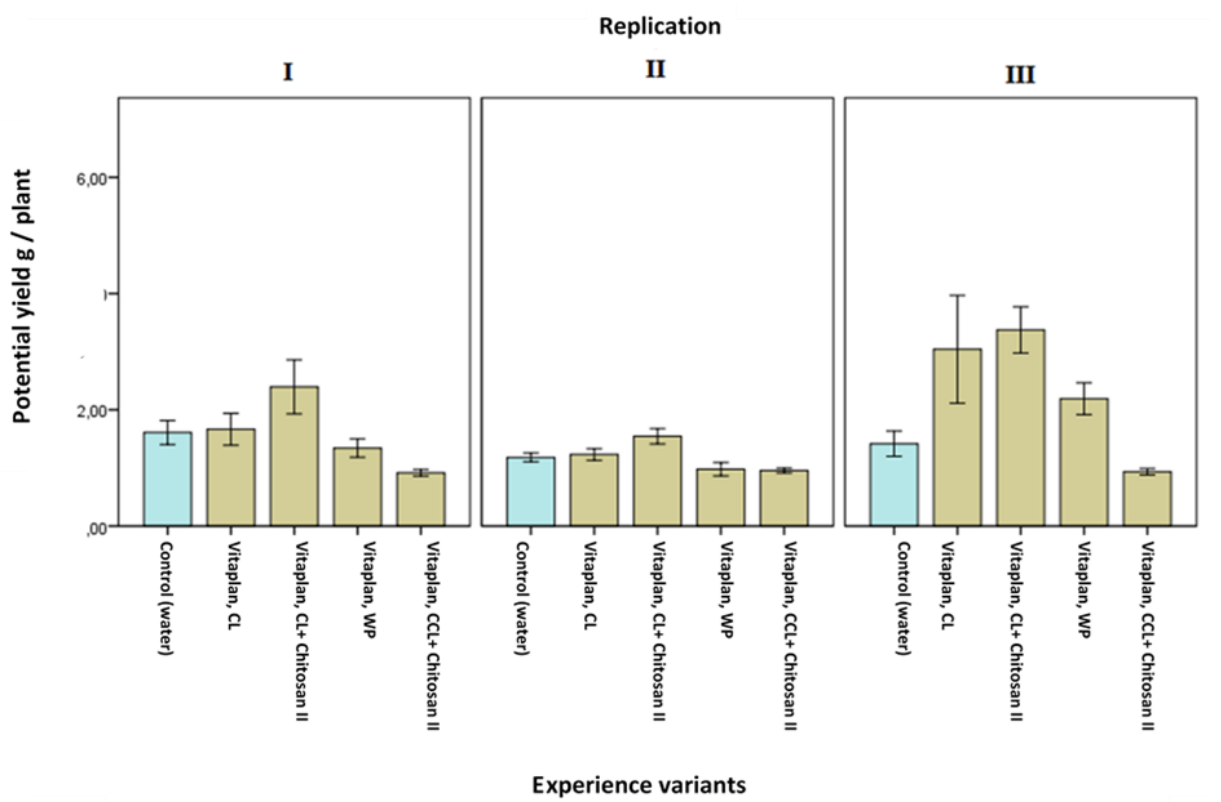

Fig. 2. - Potential yield of soft wheat after using biopreparations and polyfunctional complexes $(95 \%$ confidence intervals for means). SPb. - Pushkin, 2016-2018.

The factor analysis method established a strong correlation between potential yield and productive tilling capacity. The largest value of productive tilling capacity was found in the "Vitaplan, CL and Chitosan II" treatment. Potential wheat yields also positively correlated with root weight and flag leaf area. Maximum root weight values were recorded in the case of Vitaplan, CL and Chitosan II polyfunctional complex treatments. 
The cluster analysis method (k-medium) revealed 3 groups of indicators characterizing wheat productivity and differing in relative changes compared to the control. Validation of cluster analysis results is confirmed by variance analysis method.

It was found that the Vitaplan, CL and Chitosan II complex application increased productive tilling capacity (by $80 \%, t=7,6$ ), flag leaf area (by $30 \%, t=2,4$ ) and root weight (by $20 \%$, $\mathrm{t}=2,3)$.

The application of the Vitaplan, CL and Chitosan II polyfunctional complex significantly increased the rate of plant development (by the phases of ontogenesis) - by $10 \%(\mathrm{t}=2,4)$ and their height - by $25 \%(t=2,8)$ for the period of 2016-2018.

Spectrometric assessment of soft wheat crops found that the application of the "Vitaplan, CL, CCL and Chitosan II" polyfunctional complexes strongly reduced the plant stress index (NDVI), which is a relative indicator of the amount of photosynthetically active biomass (Fig. 3).

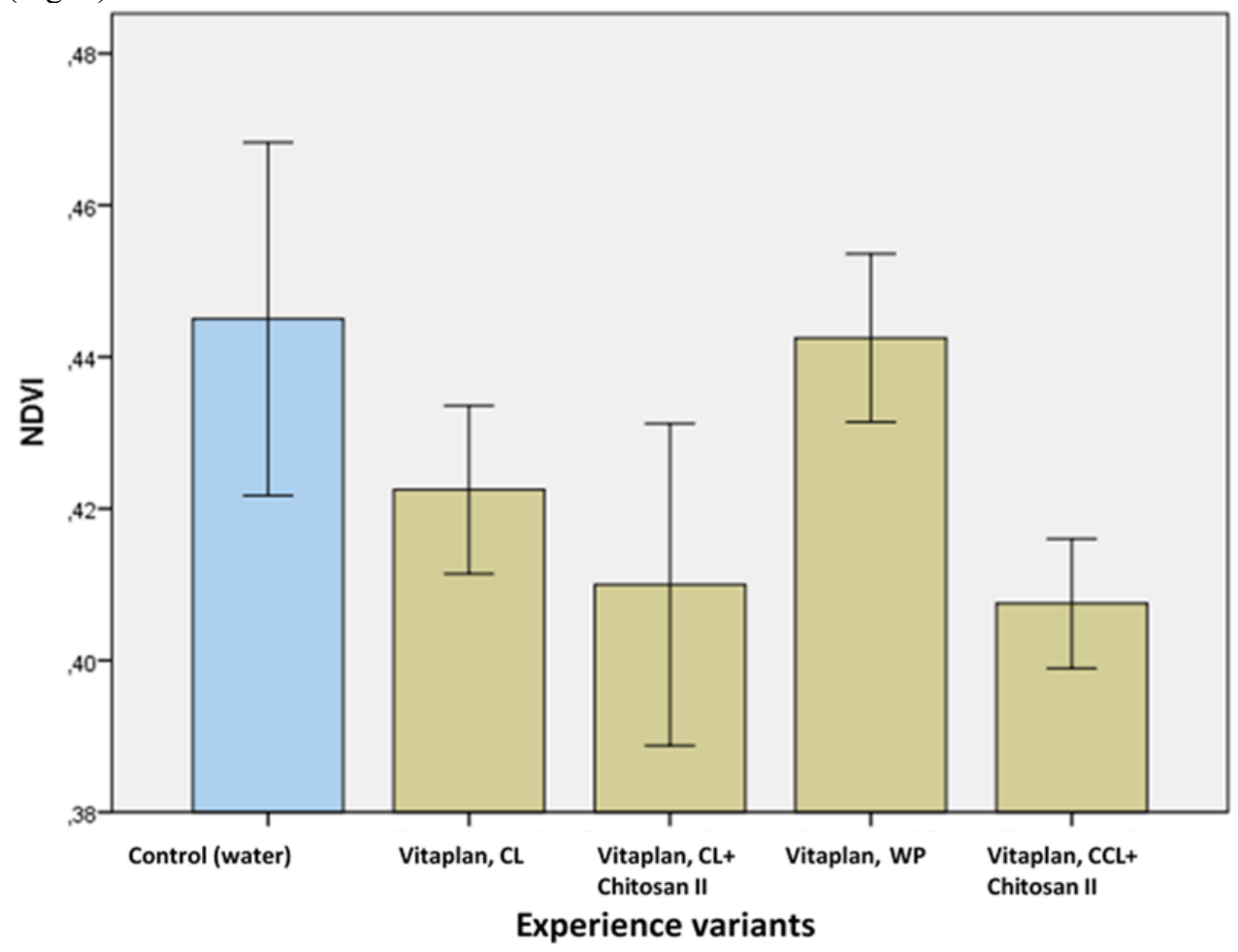

Fig. 3. Soft wheat stress index after using biopreparations and polyfunctional complexes ( $95 \%$ confidence intervals for means).

Stress index values correlated inversely with seed germination in field, as well as the number and length of node roots. Increased intensity of root rot and brown rust development, on the contrary, led to an increase in stress index values.

Thus, as a result of the research carried out, high biological efficiency of polyfunctional biopreparations showing the reduction of disease intensity and the increase of wheat productivity was noted. Pathogenesis indicators were reduced significantly as compared to the control in the "Vitaplan, CCL and Chitosan II" test variant, and the application of the "Vitaplan, CL and Chitosan II" polyfunctional complex resulted in the highest increase in potential wheat yield. The research has shown that the application of the Vitaplan, CL and 
Chitosan II, Vitaplan, CCL and Chitosan II polyfunctional complexes resulted in the most pronounced reduction of plant stress index (NDVI), which indicates the possibility of its adoption as an integral indicator in the analysis of biological efficiency of polyfunctional biopreparations.

\section{References}

1. V.A. Pavlyushin, I.I. Novikova, S.L. Tyuterev, E.V. Popova, G.A. Bykova, N.S Domnina, Biotechnol., 4, 69-80 (2010)

2. I.I. Novikova, Yu.A. Titova, I.V. Boykova, V.N. Zeyruk, I.L. Krasnobaeva, T.A. Serova, Plant Protection News [Vestnik zashchity rasteniy], 3(93), 16-23 (2017)

3. The State catalog of pesticides and agrochemicals, approved for use in the Russian Federation (2019)

4. S.L. Tyuterev, Natural and synthetic inducers of plant resistance to diseases (SPb.: Ed. VIZR. 2014)

5. Compant S., Brader G., Muzammil S., Sessitsch A., Lebrihi A., Mathieu F. Use of beneficial bacteria and their secondary metabolites to control grapevine pathogen diseases. BioControl, 2013, 58, 435-455 (doi:10.1007/s10526-012-9479-6).

6. Kolesnikov L.E., Novikova I.I., Surin V.G., Popova E.V., Priyatkin N.S., Kolesnikova J.R. Applied Biochemistry and Microbiology. 2018, 54, 5:546-552.

7. A.M. Shpanev, II All-Russian Sci. Conf. Proc. "Application of remote sensing in agriculture", 379-383 (St. Petersburg, Sept. 26-28, 2018). 